\title{
Correction to: Degenerate Bernstein polynomials
}

\section{Taekyun $\mathrm{Kim}^{1} \cdot$ Dae San $\mathrm{Kim}^{2}$}

Published online: 11 March 2019

(c) The Royal Academy of Sciences, Madrid 2019

\section{Correction to: RACSAM}

\section{https://doi.org/10.1007/s13398-018-0594-9}

Unfortunately, erratua appear in the statement corresponding Theorems 2.6 and 2.10 in the original paper. In the statemets of the theorems, the identities must be corrected as follows. After this modification, the amended proofs are also provided.

Theorem 2.6 For $n, i \in \mathbb{N}$, with $i \leq n$, and $x \in[0,1]$, we have

$$
(x)_{i, \lambda}=\frac{1}{(1-i \lambda)_{n-i, \lambda}} \sum_{k=i}^{n} \frac{\left(\begin{array}{c}
k \\
i
\end{array}\right)}{\left(\begin{array}{c}
n \\
i
\end{array}\right)} B_{k, n}(x \mid \lambda) .
$$

Theorem 2.10 For $n, i \in \mathbb{N}$, with $i \leq n$, and $x \in[0,1]$, we have

$$
\sum_{l=0}^{i}(x)_{l} S_{2, \lambda}(i, l)=\frac{1}{(1-i \lambda)_{n-i, \lambda}} \sum_{k=i}^{n} \frac{\left(\begin{array}{c}
k \\
i
\end{array}\right)}{\left(\begin{array}{l}
n \\
i
\end{array}\right)} B_{k, n}(x \mid \lambda),
$$

where $(x)_{l}=x(x-1)(x-2) \cdots(x-l+1)$.

\section{Proof of Theorem 2.6}

$$
\begin{aligned}
\sum_{k=1}^{n} \frac{\left(\begin{array}{l}
k \\
1
\end{array}\right)}{\left(\begin{array}{c}
n \\
1
\end{array}\right)} B_{k, n}(x \mid \lambda) & =\sum_{k=1}^{n} \frac{k}{n}\left(\begin{array}{l}
n \\
k
\end{array}\right)(x)_{k, \lambda}(1-x)_{n-k, \lambda} \\
& =\sum_{k=1}^{n}\left(\begin{array}{c}
n-1 \\
k-1
\end{array}\right)(x)_{k, \lambda}(1-x)_{n-k, \lambda} \\
& =\sum_{k=0}^{n-1}\left(\begin{array}{c}
n-1 \\
k
\end{array}\right)(x)_{k+1, \lambda}(1-x)_{n-k-1, \lambda}
\end{aligned}
$$

The original article can be found online at https://doi.org/10.1007/s13398-018-0594-9.

$\bowtie \quad$ Taekyun Kim

tkkim@kw.ac.kr

Dae San Kim

dskim@sogang.ac.kr

1 Department of Mathematics, Kwangwoon University, Seoul 139-701, Republic of Korea

2 Department of Mathematics, Sogang University, Seoul 121-742, Republic of Korea 


$$
\begin{aligned}
& =(x)_{1, \lambda} \sum_{k=0}^{n-1}\left(\begin{array}{c}
n-1 \\
k
\end{array}\right)(x-\lambda)_{k, \lambda}(1-x)_{n-k-1, \lambda} \\
& =(x)_{1, \lambda}(1-\lambda)_{n-1, \lambda} .
\end{aligned}
$$

Now, we observe that

$$
\begin{aligned}
\sum_{k=2}^{n} \frac{\left(\begin{array}{c}
k \\
2
\end{array}\right)}{\left(\begin{array}{c}
n \\
2
\end{array}\right)} B_{k, n}(x \mid \lambda) & =\sum_{k=2}^{n} \frac{k(k-1)}{n(n-1)}\left(\begin{array}{l}
n \\
k
\end{array}\right)(x)_{k, \lambda}(1-x)_{n-k, \lambda} \\
& =\sum_{k=2}^{n}\left(\begin{array}{l}
n-2 \\
k-2
\end{array}\right)(x)_{k, \lambda}(1-x)_{n-k, \lambda} \\
& =\sum_{k=0}^{n-2}\left(\begin{array}{c}
n-2 \\
k
\end{array}\right)(x)_{k+2, \lambda}(1-x)_{n-k-2, \lambda} \\
& =(x)_{2, \lambda} \sum_{k=0}^{n-2}\left(\begin{array}{c}
n-2 \\
k
\end{array}\right)(x-2 \lambda)_{k, \lambda}(1-x)_{n-k-2, \lambda} \\
& =(x)_{2, \lambda}(1-2 \lambda)_{n-2, \lambda} .
\end{aligned}
$$

Similarly, we have

$$
\sum_{k=i}^{n} \frac{\left(\begin{array}{l}
k \\
i
\end{array}\right)}{\left(\begin{array}{c}
n \\
i
\end{array}\right)} B_{k, n}(x \mid \lambda)=(x)_{i, \lambda}(1-i \lambda)_{n-i, \lambda}
$$

Proof of Theorem 2.10 For $n \geq 0$, the degenerate Stirling number of the second kind is given by

$$
(x)_{n, \lambda}=\sum_{k=0}^{n} S_{2, \lambda}(n, k)(x)_{k} .
$$

Thus, by Theorem 2.6, we get

$$
\sum_{l=0}^{i}(x)_{l} S_{2, \lambda}(i, l)=\frac{1}{(1-i \lambda)_{n-i, \lambda}} \sum_{k=i}^{n} \frac{\left(\begin{array}{c}
k \\
i
\end{array}\right)}{\left(\begin{array}{c}
n \\
i
\end{array}\right)} B_{k, n}(x \mid \lambda) .
$$

Publisher's Note Springer Nature remains neutral with regard to jurisdictional claims in published maps and institutional affiliations. 